\title{
Multidetector computed tomography assessment of vascular involvement in perihilar cholangiocarcinoma
}

\author{
Lotte C. Franken ${ }^{1}$, Robert J. S. Coelen ${ }^{1}$, Joris I. Erdmann ${ }^{1}$, Joanne Verheij ${ }^{2}$, Marnix P. Kop ${ }^{3}$, \\ Thomas M. van Gulik ${ }^{1}$, Saffire S. Phoa ${ }^{3}$ \\ ${ }^{1}$ Department of Surgery, Cancer Center Amsterdam, Amsterdam UMC, University of Amsterdam, Amsterdam, The Netherlands; ${ }^{2}$ Department of \\ Pathology, Amsterdam UMC, University of Amsterdam, Amsterdam, The Netherlands; ${ }^{3}$ Department of Radiology, Amsterdam UMC, University of \\ Amsterdam, Amsterdam, The Netherlands
}

Contributions: (I) Conception and design: TM van Gulik, SS Phoa; (II) Administrative support: LC Franken, RJS Coelen, MP Kop; (III) Provision of study materials or patients: MP Kop, SS Phoa, JI Erdmann, TM van Gulik, J Verheij; (IV) Collection and assembly of data: LC Franken, RJS Coelen, MP Kop, SS Phoa; (V) Data analysis and interpretation: LC Franken, RJS Coelen, MP Kop, SS Phoa, TM van Gulik; (VI) Manuscript writing: All authors; (VII) Final approval of manuscript: All authors.

Correspondence to: Saffire S. Phoa, Radiologist. Amsterdam UMC, Meibergdreef 9, 1105 AZ, Amsterdam, The Netherlands.

Email: s.s.phoa@amsterdamumc.nl.

Background: In approximately $40 \%$ of patients with perihilar cholangiocarcinoma (PHC), the tumor is deemed unresectable at laparotomy, often due to vascular involvement. On imaging, occlusion, narrowing, wall irregularity and $>180^{\circ}$ tumor-vessel contact have been suggested to predict vascular involvement in patients with PHC. The objective of this study was to correlate computed tomography (CT) findings in PHC with surgical and histopathological results, in order to evaluate the accuracy of currently used CT criteria for vascular involvement.

Methods: Patients with PHC undergoing exploration in a single tertiary center (2015-2018) were included. Tumor-vessel relation of portal vein and hepatic artery on CT were scored by two independent radiologists, blinded for surgical and pathological outcomes. Intraoperative findings were scored by the surgeon in theatre or derived from operation/pathology reports.

Results: A total of 42 CT scans were evaluated, resulting in assessment of 115 vessels. Portal vein occlusion, narrowing and presence of an irregular wall on CT corresponded with a positive predictive value (PPV) for involvement of $100 \%, 83 \%$ and $75 \%$, respectively. For the hepatic artery, PPV of occlusion and stenosis was $100 \%$, whilst other criteria had PPV $<70 \%$. Combining potential criteria $\left(>180^{\circ}\right.$ contact, narrowing, irregularity or occlusion) resulted in PPV, sensitivity and specificity of $85 \%, 67 \%$ and $94 \%$, respectively, for the portal vein and 53\%, 40\% and 75\%, respectively, for the hepatic artery.

Conclusions: Prediction of vascular involvement on CT is more difficult for the hepatic artery than for the portal vein. Suggestion of hepatic artery invasion on imaging, other than occlusion or stenosis, should not preclude surgical exploration.

Keywords: Perihilar cholangiocarcinoma (PHC); vascular involvement; computed tomography (CT); hepatic artery; portal vein; resectability

Submitted Nov 25, 2020. Accepted for publication Apr 27, 2021.

doi: 10.21037 /qims-20-1303

View this article at: https://dx.doi.org/10.21037/qims-20-1303 


\section{Introduction}

Perihilar cholangiocarcinoma (PHC) is a relatively rare disease with an annual incidence of 200 patients in the Netherlands (1). Radical surgery is the only potentially curative treatment, often consisting of resection of the extrahepatic bile duct and hemihepatectomy (2). To date, no accurate widely implemented staging system exists to assess resectability (3-5). In daily practice, resectability is determined in a multidisciplinary meeting. Preoperative imaging to define the extent of the biliary tumor is preferably performed before biliary drainage and often includes a combination of computed tomography (CT) and magnetic resonance imaging or cholangiography (MRI/ MRCP).

For assessment of resectability multiphase contrastenhanced CT (CECT) is regarded to be superior compared to MRI to demonstrate the relationship of the tumor to vascular structures (6). A systematic review with metaanalysis from our group showed a pooled sensitivity of CECT for portal vein and hepatic artery involvement of $89 \%$ and $84 \%$, respectively (7). In literature, suggested CT criteria for vascular involvement are occlusion, stenosis or contour deformity and more than $50 \%$ contact with the tumor $(6,8)$.

Currently, approximately $40 \%$ of patients with potentially resectable $\mathrm{PHC}$ on imaging are deemed unresectable at explorative laparotomy. The most important reason for unresectability at exploration is extensive portal venous and/or hepatic arterial vascular involvement, as assessed intraoperatively by the surgeon and often confirmed by frozen section. It is clear that vascular involvement and consequent unresectability are still largely underestimated by imaging preoperatively $(9,10)$. This not only leads to unnecessary surgery, with subsequent lower patient quality of life and high costs, but also has major implications for treatment strategies in PHC patients. Accurate assessment of resectability on imaging is the cornerstone of selecting the right patient for the right treatment.

Therefore, the aim of this study was to correlate CT findings in PHC with surgical and histopathological results, in order to evaluate the accuracy of currently used criteria for vascular involvement on preoperative CECT imaging.

\section{Methods}

All patients who underwent surgical exploration under the suspicion of PHC between March 2015 and April 2018 at one tertiary center were included in this study.

The study was conducted in accordance with the Declaration of Helsinki (as revised in 2013). The study was approved by institutional ethics board of the Amsterdam UMC, location AMC (W14_328) and individual consent for this retrospective analysis was waived.

Standard work-up of patients with $\mathrm{PHC}$ at our institution has previously been described in detail (11), and includes a preoperative staging CT scan. In our institution, concomitant resection of the portal vein bifurcation is only performed when the portal vein is involved. Standard CT protocol included a dynamic contrast-enhanced CT scan with arterial and venous phase. Three scanners were used, with the following specifications: Siemens Force CT scanner, AS+, Sensation (multi-slice 64, 122, 192), collimation $0.6 \mathrm{~mm}$, reconstruction 2 and $3 \mathrm{~mm}$, late arterial phase (with bolus timing peak $+30 \mathrm{~s}$ ) and portal venous phase (70 s fixed), Ultravist $100-130 \mathrm{~mL}(3.5 \mathrm{~mL} / \mathrm{s})$. Coronal and sagittal reconstructions were performed and maximum intensity projections (MIP) were not used. The most recent CT scan prior to surgery (all within 6 weeks) was independently assessed by two dedicated radiologists, who were blinded for clinical information, other imaging and outcome.

Vascular contact of the hepatic artery and portal vein was evaluated, including their main trunk and right and left branches. The relation between the tumor and the vessel was assessed by scoring circumference of involvement, length of contact and the presence of irregular wall, stenosis and occlusion. For the portal vein the circumferential involvement was assessed as $<90^{\circ}, 90^{\circ}-180^{\circ}, 180^{\circ}-270^{\circ}$, $>270^{\circ}$. For the hepatic artery the circumferential involvement was assessed as the presence of tumor on one or two sides of hepatic artery $\left(<180^{\circ}\right.$ or $\left.>180^{\circ}\right)$, since scoring the precise circumferential involvement is difficult for (small) arteries. The length of contact was scored as less than $10 \mathrm{~mm}, 10-20 \mathrm{~mm}$ or more than $20 \mathrm{~mm}$. Discrepancies were resolved by a consensus meeting and recorded as a third assessment. In case both radiologists individually evaluated a scan not assessable due to bad quality of the scan or substantial artifacts-especially present when portal vein embolization material was in situ-these patients were excluded. Severity of artefacts differed between patients; therefore not all patients undergoing PVE were excluded.

Radiological findings were compared to intraoperative findings. Intraoperative findings were obtained from the operation report or scored by the surgeon in theatre. Vascular involvement was defined as infiltration of the vessel by the tumor, not separable by the surgeon at dissection 


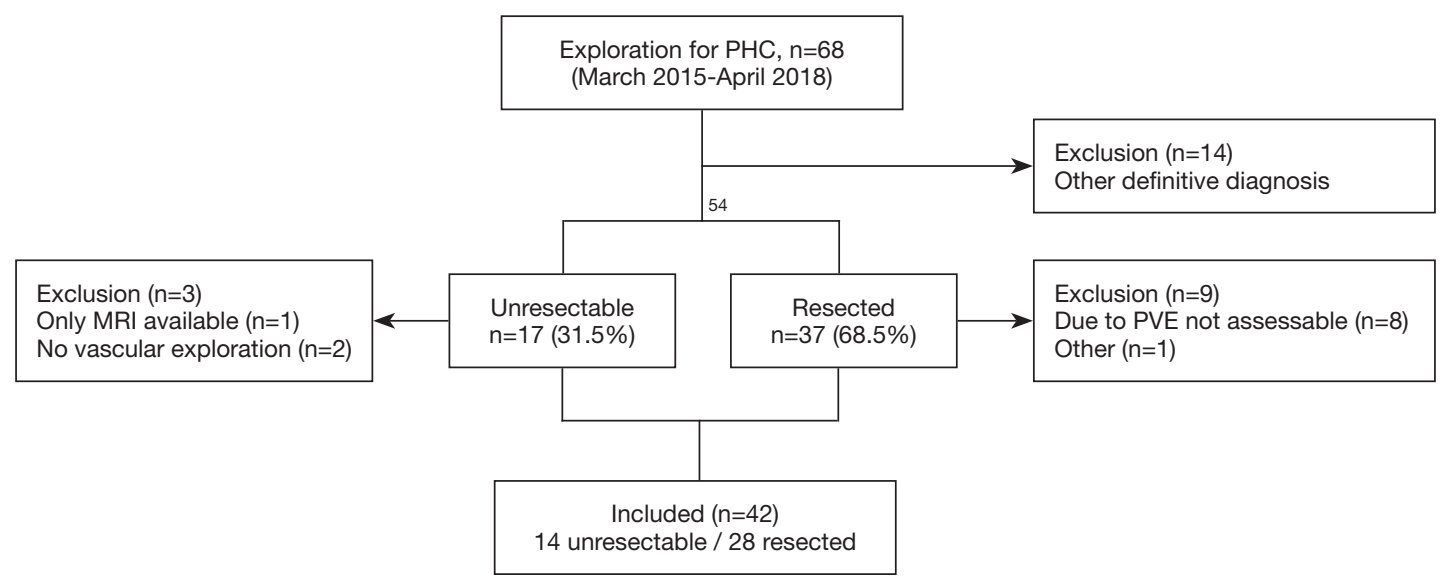

Figure 1 Flowchart of all included patients. PHC, perihilar cholangiocarcinoma; PVE, portal vein embolization; MRI, magnetic resonance imaging.

and often confirmed by frozen-section biopsy. Tumorvessel contact was defined as tumor making contact with the vessel, but easily separable from the tumor. Vascular involvement was assessed intraoperatively by the surgeon, since this assessment determines resectability. However, pathological findings were also obtained from the pathology report, including intraoperative frozen-sections and, in case of resection, histopathological evaluation of the surgical specimen. Assessment of resection planes of the hepatic artery and portal vein were used to confirm surgical findings.

\section{Statistical analysis}

Statistical analysis was performed using SPSS version 25 software (IBM, Armonk, New York, USA). Descriptive statistics were used to describe the data. Variables that followed a normal distribution were described by mean and standard deviation, whilst variables that did not follow normal distribution were described by median with interquartile range. Negative and positive predicting values, sensitivity and specificity for vascular involvement were calculated for separate and combined criteria. For prediction of involvement, intraoperative tumor-vessel contact that could be easily separated by the surgeon was scored as an uninvolved vessel (since this did not preclude resection). The interobserver variability was assessed by calculating Cohen's kappa.

\section{Results}

A total of 68 patients underwent explorative laparotomy for suspicion of PHC, of which 14 patients (20\%) ultimately had a different diagnosis at final pathological examination. Of the remaining 54 pathologically proven $\mathrm{PHC}$-patients, preoperative CT scans of 42 patients were evaluated. Twelve patients were excluded from analysis, with severe artefacts from portal vein embolization material as the most frequent reason for exclusion (as shown in Figure 1). There were 20 males and 22 females and the mean age was $68 \pm 7$ years. The distribution of patients with Bismuth-Corlette (based on surgical findings) type II, IIIa, IIIb, IV was 3, 20, 5 and 14 patients, respectively. The median time between CT scan and operation was 20 days. Out of 42 patients, there were 28 patients with biliary stents in situ (including 9 percutaneous, 11 endoscopic, 7 percutaneous + endoscopic and 1 wall stent) and 14 patients without biliary drainage with stents prior to the assessed CT scan.

\section{Unresectability}

Out of 54 patients with pathologically proven PHC, there were 17 patients with unresectable tumors $(32 \%)$ at explorative laparotomy (Figure 1). Three of these patients were excluded due to the lack of vascular exploration $(n=2)$ or because there was only a MRI scan available $(n=1)$. Reasons for unresectability in these three patients were liver metastases $(n=1)$ and lymph node metastases $(n=2)$. Reasons for unresectability for the remaining 14 patients with unresectable disease included in the study were liver metastases $(n=2)$, lymph node metastases $(n=2)$, lymph node metastases/peritoneal metastases combined with vascular involvement $(\mathrm{n}=3)$ and/or vascular involvement $(\mathrm{n}=7)$. Table 1 
Table 1 Radiological findings of patients with unresectable PHC based on vascular involvement

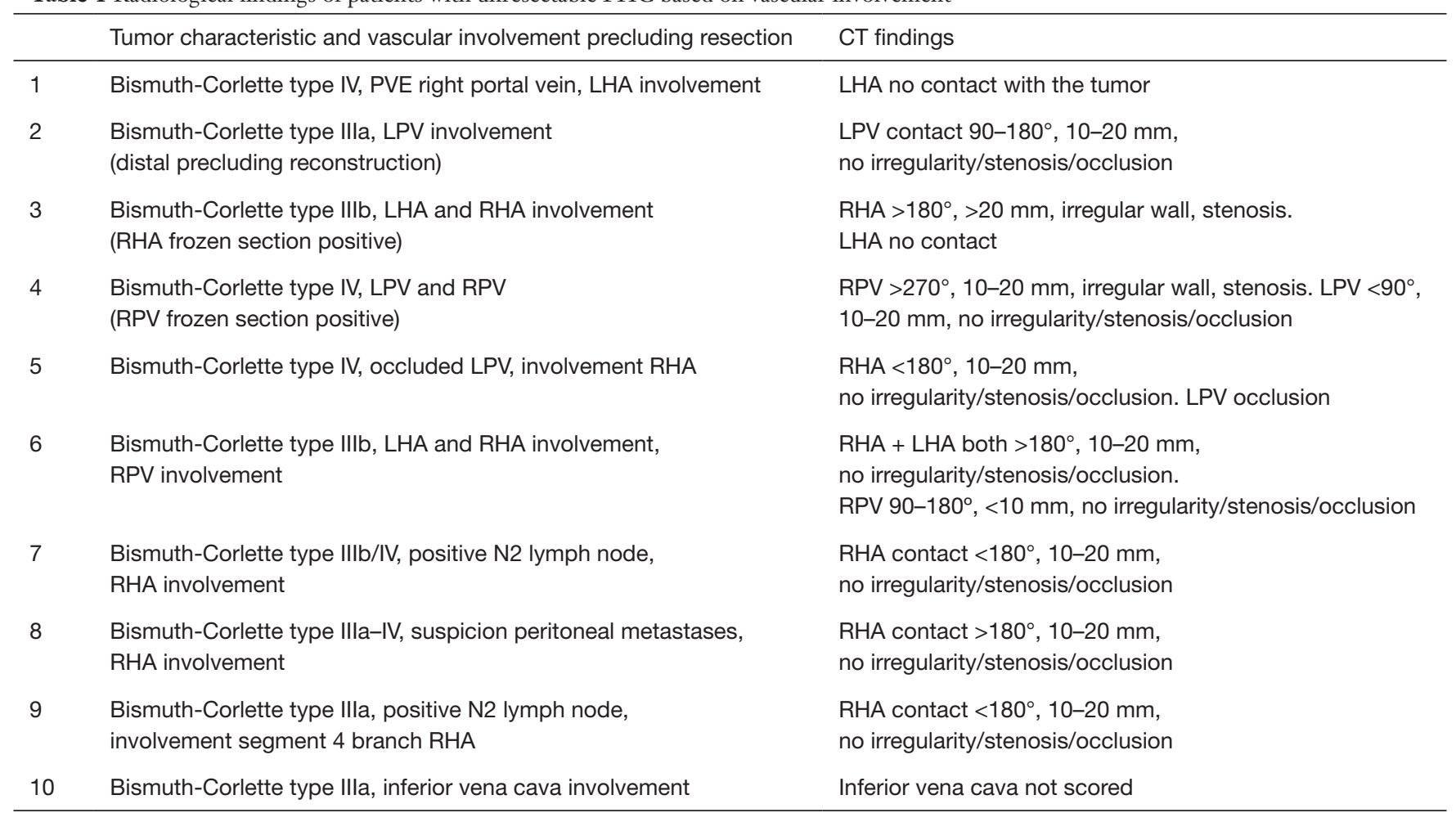

CT, computed tomography; PVE, portal vein embolization; LHA, left hepatic artery; RHA, right hepatic artery; LPV, left portal vein; RPV, right portal vein.

shows radiological findings of patients with unresectable tumors based on vascular involvement.

\section{Intraoperative findings}

Radiological findings of 42 patients with pathologically proven PHC (14 unresectable, 28 resected) were correlated to intraoperative findings. As in each patients the left and right hepatic artery and portal vein were assessed, there were a total of 168 vessels to evaluate. After exclusion of the vessels that were deemed not assessable $(n=53)$, intraoperative findings of 115 vessels could be correlated with preoperative imaging; 63 hepatic arteries and 52 portal veins. Of these vessels, there were 36 of 115 (31\%) vessels involved at intraoperative assessment: 20 hepatic arteries and 16 portal veins. There were 79 vessels that were not involved at intraoperative evaluation: at radiological consensus $47(59 \%)$ of these vessels were assessed as no contact, whilst $32(41 \%)$ of these vessels were scored as tumor-vessel contact. Figure 2 shows an example of false positive assessment of right hepatic artery involvement on CT. Out of 36 vessels that were involved at intraoperative evaluation, there were two vessels (6\%) scored as no tumorvessel contact by the radiologists.

\section{CT-criteria}

Table 2 shows the negative predictive value (NPV) and positive predictive value (PPV) of separate and combined CT criteria. Occlusion of portal vein on imaging had a PPV of $100 \%$, however it was found in only 5 of 16 involved portal veins and therefore has a low sensitivity (31\%). Likewise, the PPV for occlusion of the hepatic artery was $100 \%$, however present in only 3 of 20 involved hepatic arteries $(15 \%)$. NPV of no tumor-vessel contact for the hepatic artery and portal vein were $100 \%$ and $94 \%$, respectively. A total of 13 portal veins and 15 hepatic arteries met the criteria suggested in literature (e.g., presence of tumor-vessel contact $>180^{\circ}$ or irregular wall or narrowing or occlusion). Combination of these criteria resulted in a PPV of $85 \%$ for the portal vein, with sensitivity of $67 \%$ and specificity of $94 \%$. For the hepatic artery these combined criteria resulted in a PPV of $53 \%$, sensitivity of $40 \%$ and specificity of $86 \%$. 

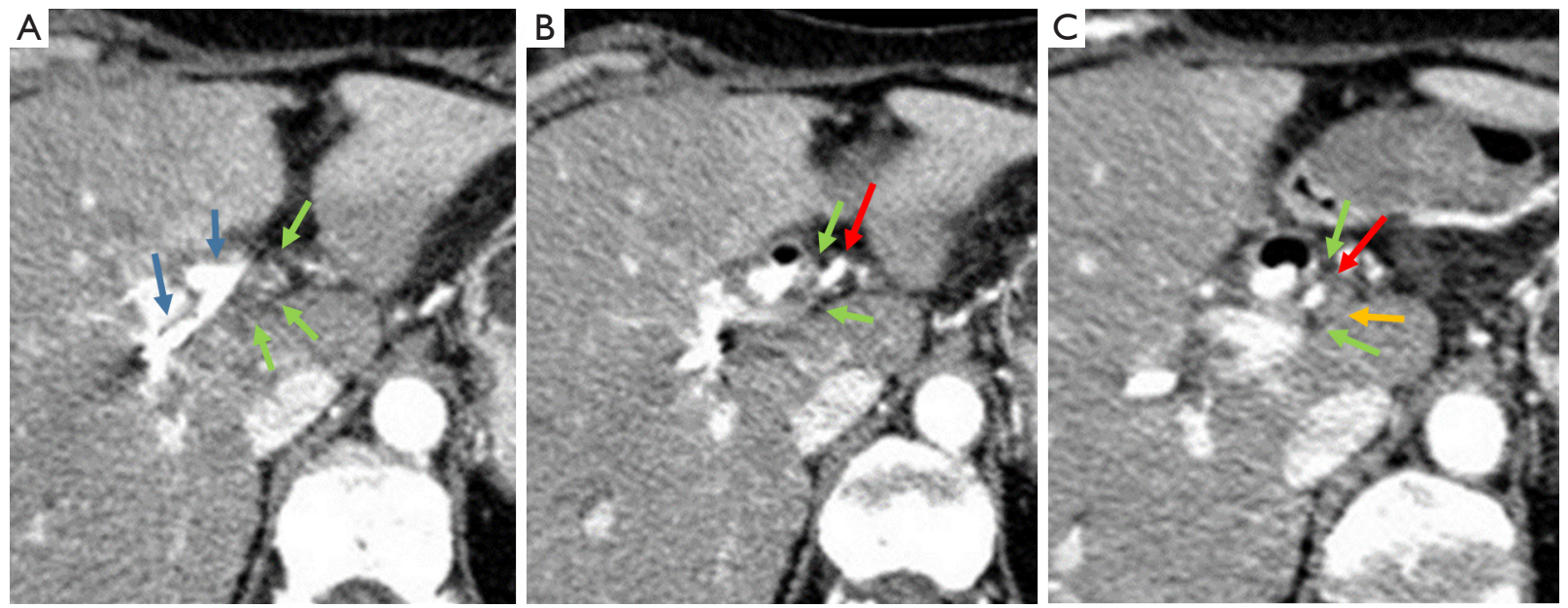

Figure 2 Arterial phase CT scan: false positive right hepatic artery (RHA) involvement. 72-year-old patient with Klatskin type 3A tumor. The RHA was judged as being involved $>180$ degrees (tumor on both sides of the artery). Although assessed as tumor on both sides of the right hepatic artery $\left(>180^{\circ}\right)$ on CT, during surgical exploration the right hepatic artery was closely related to the tumor, but was easily separable, and a left hemihepatectomy was performed. Green arrow: regarded as tumor, partially obscured by artefacts caused by the biliary stent. Red arrow: right hepatic artery. Blue arrow: Biliary stent in the right anterior and posterior bile ducts. Orange arrow: small lymph node on the left posterior side of the artery.

\section{Interobserver agreement}

A consensus meeting was necessary for 40 out 42 scans. Overall, Cohens kappa for presence or absence of contact with the vessel was 0.265 (SE 0.062). If both radiologists scored tumor-vessel contact $(n=47)$, Cohen's kappa for circumference of contact $\left(<180^{\circ},>180^{\circ}\right)$, length of contact $(<10,10-20,>20 \mathrm{~mm})$ and the presence or absence of irregular wall were 0.640 (SE 0.121), 0.374 (SE 0.122) and 0.115 (SE 0.125), respectively. For the presence of stenosis or occlusion, the Cohens kappa was 0.601 (SE 0.111) and 0.887 (SE 0.110), respectively.

\section{Discussion}

In this study, vascular involvement of PHC was investigated by correlating findings of two independent radiologists, who were blinded for surgical and pathological outcomes, with intraoperative findings. CT scans of $42 \mathrm{PHC}$-patients undergoing exploration were included and 115 vessels were evaluated. The PPVs of an occluded, narrowed or irregular portal vein were $100 \%, 83 \%$ and $75 \%$, respectively. If no tumor-vessel contact of portal vein was noted on the CT scan, this correlated with no involvement at intraoperative assessment (NPV of $100 \%$ ). For the hepatic artery, occlusion or stenosis of the vessel on imaging also resulted in a PPV of $100 \%$. Interestingly, other criteria, especially for the hepatic artery, seemed of limited value in predicting vascular involvement. Due to the smaller diameter and the winding course of the hepatic artery, assessment may be more difficult compared to the portal vein.

In literature, there are several studies focusing on prediction of vascular involvement in $\mathrm{PHC}$ on imaging. In a study published by Lee et al. in 2006 (8), with a study design comparable to ours, a PPV for portal vein involvement on CT of $91 \%$ was found. In this study, vascular involvement on transverse imaging was defined as either tumor-vessel contact more than $180^{\circ}$ or irregular wall or narrowing or occlusion of the vessel. Application of these combined CT criteria in the present study resulted in a PPV for the portal vein of $84 \%$. For the hepatic artery, however, Lee et al. (8) reported a PPV of $95 \%$, whilst in our study the PPV for the hepatic artery was $53 \%$. A factor that could have contributed to this difference may be the selection of patients for explorative laparotomy. One could imagine that assessment of extensive vascular involvement is relatively easier than the assessment of limited vascular involvement. In the study by Lee et al. (8), tumors were deemed unresectable in 32 out of 55 patients (58\%), compared to 17 tumors in 54 patients (32\%) in the present study (8).

In 2018, Zhou et al. (12) investigated several CT criteria to assess portal vein involvement in PHC. Their proposed 
Table 2 CT findings correlated with intraoperative findings. Separate and combined CT criteria with negative predictive value (NPV) and positive predictive value (PPV)

\begin{tabular}{|c|c|c|c|c|}
\hline & $\begin{array}{c}\text { Intraoperative } \\
\text { vascular invasion }\end{array}$ & $\begin{array}{l}\text { Intraoperative no } \\
\text { vascular invasion }\end{array}$ & NPV & PPV \\
\hline \multicolumn{5}{|l|}{ Portal vein (PV) on imaging ${ }^{*}$} \\
\hline PV contact $<90^{\circ}$ & 1 & 9 & 0.9 & \\
\hline PV contact $>270^{\circ}$ & 2 & 0 & & 1 \\
\hline $\mathrm{PV}<10 \mathrm{~mm}$ & 2 & 3 & 0.6 & \\
\hline PV 10-20 mm & 6 & 14 & & 0.3 \\
\hline PV occlusion & 5 & 0 & & 1 \\
\hline Combined (PV contact $>180^{\circ}$ or irregular or narrowed or occluded) & 11 & 2 & & 0.85 \\
\hline \multicolumn{5}{|l|}{ Hepatic artery $(\mathrm{HA})$ on imaging ${ }^{\star \star}$} \\
\hline No tumor-vessel contact HA & 2 & 31 & 0.94 & \\
\hline HA contact one side & 11 & 4 & 0.27 & 0.73 \\
\hline HA contact two sides & 7 & 7 & & 0.5 \\
\hline $\mathrm{HA}<10 \mathrm{~mm}$ & 2 & 3 & 0.6 & \\
\hline Combined (HA contact $>180^{\circ}$ or irregular or narrowed or occluded) & 8 & 7 & & 0.53 \\
\hline
\end{tabular}

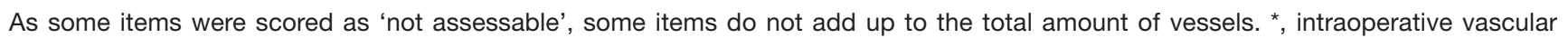
invasion $(n=16)$, intraoperative no vascular invasion $(n=36) .{ }^{*}$, intraoperative vascular invasion $(n=20)$, intraoperative no vascular invasion $(n=43)$. PV, portal vein; HA, hepatic artery.

new model included more than 180 degrees of contact and showed a PPV of $92 \%$. Interestingly, the interobserver agreement was high with kappa's between 0.870 and 0.957 . Results of both previously mentioned studies suggest that length of tumor-vessel contact of the portal vein should also be incorporated in assessment of portal vein involvement. In the first study, revised criteria include a cut-off of $2 \mathrm{~cm}(8)$, whilst the second study by Zhou et al. suggests a cut-off of $7.29 \mathrm{~mm}$ for portal vein involvement (12). In the present study, more than $2 \mathrm{~cm}$ tumor-vessel contact of the portal vein resulted in relatively low PPV of $50 \%$ and was not incorporated in the combined criteria.

Another study assessing resectability of PHC, showed a PPV of portal vein and hepatic artery involvement on CT in 31 patients of $90 \%$ and $94 \%$, respectively (13). Vascular involvement was also defined as more than 180 degrees of contact, occlusion, stenosis or contour deformity. The mean interval between CT and surgery was 10.6 days, compared to 
a mean interval of 26 days in the present study. Disagreements between two radiologists were resolved by consulting a senior hepatobiliary surgeon, which may have influenced results. Evaluation of technical specifications of different studies, revealed no explanation for the observed differences $(8,12,13)$.

Unfortunately, vascular involvement can only be evaluated in patients undergoing exploration. The inability to evaluate vessels in initially unresectable patients is one of the major limitations of this study. Even for patients undergoing resection, the assessment of vascular involvement remains challenging for pathologists $(14,15)$. As shown in literature, assessment of all relevant resection planes in PHC is often incomplete and the hepatic artery resection plane is most often lacking. Pathological parameters that are not described are often regarded as absent rather than as missing $(14,15)$.

Complete pathological assessment of the relevant vessel is not always possible, since the surgical specimen is laminated following the biliary tree. As a consequence, the nature of the vessels seen in the pathology specimen (main hilar vessels or subsequent arterial and venous branches) is not always clear and the correlation between imaging and pathological assessment may be very difficult. Additionally, it remains unclear which layer (if infiltrated) of the vascular wall (adventitia, media and endothelium) defines relevant vascular involvement. In previous studies correlating pathological findings to CT images, these difficulties in pathological assessment may also have affected results $(8,12,13)$.

Other limitations involve the retrospective evaluation of the majority of patients in this study, the lack of an optimal arterial reconstruction in some patients and the small sample size. Also, in this study the most recent CT scan prior to surgery was evaluated without access to other imaging modalities such as MRCP or previous scans, which could have influenced evaluation by the radiologists. Infiltration after interventions such as stenting and portal vein embolization could also influence assessment of vascular invasion (Figure S1). However, blinded evaluation by two independent radiologists of a single CT scan limited the risk of bias and is one of the strengths of this study. Additionally, we evaluated all consecutive patients explored in one institution in a recent period, making results translatable to current daily practice.

Although these results should be interpreted with caution considering limitations of this study, the observed discrepancies between imaging and intraoperative findings have major impact on clinical decision making and treatment strategies (16). If, for example, prediction of involvement only seems reliable in case of encasement or occlusion of the hepatic artery, other criteria (such as more than 180 degrees or a certain length of contact) should not preclude exploration. If assessment on imaging is indeed unreliable, explorative laparotomy might be necessary to prove 'locally advanced disease'. This would not only lead to more unnecessary explorations, but specifically for PHC patients with locally advanced tumors would also eliminate the possibility of liver transplantation, since surgical exploration of the hepatoduodenal ligament is a contraindication in the (Dutch) liver transplantation protocol (17). On the other hand, patients (falsely) deemed unresectable based on imaging, could potentially have been denied an explorative laparotomy. The lack of resectability criteria also hampers the design of clinical trials. For example, studies on neoadjuvant or induction chemotherapy require solid resection criteria to determine a subgroup of patients with 'locally advanced disease' that may benefit from this treatment.

In conclusion, the assessment of vascular involvement of PHC on CT remains challenging. An occluded, irregular or narrowed portal vein is highly suggestive for venous invasion. CT criteria for hepatic artery involvement, other than stenosis or occlusion, are less reliable and therefore may not preclude exploration in patients with PHC.

\section{Acknowledgments}

Funding: None.

\section{Footnote}

Conflicts of Interest: All authors have completed the ICMJE uniform disclosure form (available at https://dx.doi. org/10.21037/qims-20-1303). The authors have no conflicts of interest to declare.

Ethical Statement: The authors are accountable for all aspects of the work in ensuring that questions related to the accuracy or integrity of any part of the work are appropriately investigated and resolved. The study was conducted in accordance with the Declaration of Helsinki (as revised in 2013). The study was approved by institutional ethics board of the Amsterdam UMC, location AMC (W14_328) and individual consent for this retrospective analysis was waived.

Open Access Statement: This is an Open Access article distributed in accordance with the Creative Commons 
Attribution-NonCommercial-NoDerivs 4.0 International License (CC BY-NC-ND 4.0), which permits the noncommercial replication and distribution of the article with the strict proviso that no changes or edits are made and the original work is properly cited (including links to both the formal publication through the relevant DOI and the license). See: https://creativecommons.org/licenses/by-nc-nd/4.0/.

\section{References}

1. van der Geest LA. Kankerzorg in beeld. Galblaas- en proximaal galwegcarcinoom. Rapportage IKNL. 2014.

2. Ito F, Cho CS, Rikkers LF, Weber SM. Hilar cholangiocarcinoma: current management. Ann Surg 2009;250:210-8.

3. Amin MB, Edge, S, Greene F, Byrd DR, Brookland RK, Washington MK, Gershenwald JE, Compton CC, Hess KR, Sullivan DC, Jessup JM, Brierley JD, Gaspar LE, Schilsky RL, Balch CM, Winchester DP, Asare EA, Madera M, Gress DM, Meyer LR. editors. AJCC Cancer Staging Manual. 8th edition. Springer International Publishing, 2017.

4. Matsuo K, Rocha FG, Ito K, D'Angelica MI, Allen PJ, Fong Y, Dematteo RP, Gonen M, Endo I, Jarnagin WR. The Blumgart preoperative staging system for hilar cholangiocarcinoma: analysis of resectability and outcomes in 380 patients. J Am Coll Surg 2012;215:343-55.

5. Deoliveira ML, Schulick RD, Nimura Y, Rosen C, Gores G, Neuhaus P, Clavien PA. New staging system and a registry for perihilar cholangiocarcinoma. Hepatology 2011;53:1363-71.

6. Engelbrecht MR, Katz SS, van Gulik TM, Laméris JS, van Delden OM. Imaging of perihilar cholangiocarcinoma. AJR Am J Roentgenol 2015;204:782-91.

7. Ruys AT, van Beem BE, Engelbrecht MR, Bipat S, Stoker J, Van Gulik TM. Radiological staging in patients with hilar cholangiocarcinoma: a systematic review and metaanalysis. Br J Radiol 2012;85:1255-62.

8. Lee HY, Kim SH, Lee JM, Kim SW, Jang JY, Han JK, Choi BI. Preoperative assessment of resectability of hepatic hilar cholangiocarcinoma: combined CT and cholangiography with revised criteria. Radiology 2006;239:113-21.

9. Gaspersz MP, Buettner S, Roos E, van Vugt JLA, Coelen RJS, Vugts J, et al. A preoperative prognostic model to predict surgical success in patients with perihilar cholangiocarcinoma. J Surg Oncol 2018;118:469-76.

10. Wiggers JK, Groot Koerkamp B, van Klaveren D, Coelen RJ, Nio CY, Allen PJ, Besselink MG, Busch OR, D'Angelica MI, DeMatteo RP, Kingham TP, van Gulik TM, Jarnagin WR. Preoperative Risk Score to
Predict Occult Metastatic or Locally Advanced Disease in Patients with Resectable Perihilar Cholangiocarcinoma on Imaging. J Am Coll Surg 2018;227:238-246.e2.

11. Rassam F, Roos E, van Lienden KP, van Hooft JE, Klümpen HJ, van Tienhoven G, Bennink RJ, Engelbrecht MR, Schoorlemmer A, Beuers UHW, Verheij J, Besselink MG, Busch OR, van Gulik TM. Modern work-up and extended resection in perihilar cholangiocarcinoma: the AMC experience. Langenbecks Arch Surg 2018;403:289-307.

12. Zhou Q, Guan Y, Mao L, Zhu Y, Chen J, Shi J, Tang M, Qiu Y, Zhu B, He J. Modification and establishment of $\mathrm{CT}$ criteria in preoperative assessment of portal venous invasion by hilar cholangiocarcinoma. HPB (Oxford) 2018;20:1163-71.

13. Ni Q, Wang H, Zhang Y, Qian L, Chi J, Liang X, Chen T, Wang J. MDCT assessment of resectability in hilar cholangiocarcinoma. Abdom Radiol (NY) 2017;42:851-60.

14. Roos E, Franken LC, Soer EC, van Hooft JE, Takkenberg RB, Klümpen HJ, Wilmink JW, van de Vijver MJ, van Gulik TM, Verheij J. Lost in translation: confusion on resection and dissection planes hampers the interpretation of pathology reports for perihilar cholangiocarcinoma. Virchows Arch 2019;475:435-43.

15. Chatelain D, Farges O, Fuks D, Trouillet N, Pruvot FR, Regimbeau JM. Assessment of pathology reports on hilar cholangiocarcinoma: the results of a nationwide, multicenter survey performed by the AFC-HC-2009 study group. J Hepatol 2012;56:1121-8.

16. Gilbert JW, Wolpin B, Clancy T, Wang J, Mamon H, Shinagare AB, Jagannathan J, Rosenthal M. Borderline resectable pancreatic cancer: conceptual evolution and current approach to image-based classification. Ann Oncol 2017;28:2067-76.

17. Protocol - Indicatiestelling en selectie voor levertransplantatie bij patiënten met perihilair cholangiocarcinoom. Available online: https://www.umcg.nl/ NL/UMCG/Afdelingen/Levertransplantatiecentrum\%20 UMCG/professionals/Documents/Protocol\%20 Indicatiestelling\%20en\%20selectie\%20voor\%20 levertransplantatie\%20bij\%20pati\%C3\%ABnten\%20 met\%20perihilair\%20cholangiocarcinoom.pdf

Cite this article as: Franken LC, Coelen RJS, Erdmann JI, Verheij J, Kop MP, van Gulik TM, Phoa SS. Multidetector computed tomography assessment of vascular involvement in perihilar cholangiocarcinoma. Quant Imaging Med Surg 2021;11(11):4514-4521. doi: 10.21037/qims-20-1303 


\section{Supplementary}
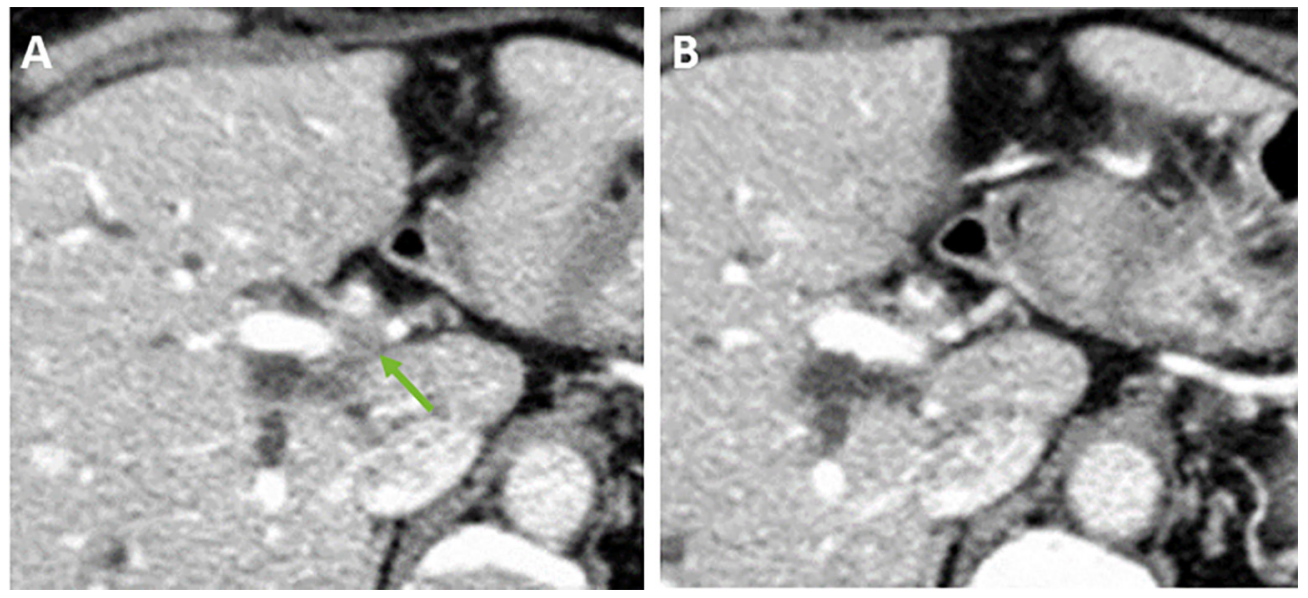

Figure S1 Portovenous phase of a CT scan prior to the CT scan in Figure 2, before stent insertion. Green arrow; regarded as tumor, better lineation of both artery and tumor. This CT scan was not available to readers, as they only assessed the most recent CT scan prior to surgery. 